\title{
The Comparative Study on VHF Radio Received Link
}

LIU Guangkai, a , QUAN Houde ${ }^{1, a}$, CUI Peizhang ${ }^{1,}$, ,YAO Shaolin ${ }^{1, \text { a }}$

${ }^{1}$ Department of Information Engineering, Mechanic Engineering College, Shijiazhuang, 050003,

China

adreamer_gk@163.com

Key words : VHF radio; the received model; filtering characteristics; GMSK demodulation

\begin{abstract}
Aiming at the research on the VHF radio received link, the received model is established. First, the filtering process, GMSK demodulation, RS decoding and de interleaving process which influence the received signal-to-noise ratio(SNR), are analyzed and modeled, so that the theoretical model is established; then, the influence of the received bit of error(BER) caused by the received link is comparatively analysis through the simulation experiment on which the received link is compared with the theoretical model; finally, the important received link is summarized.
\end{abstract}

\section{Introduction}

Some VHF radio uses the combined modulation of frequency hopping and GMSK, RS coding, interleaving multilevel coding system and multilevel filter anti-jamming measures, so that the system has characteristics of a small spectrum strays and strong anti-jamming abilities which assure the reliability of communication. In [1] the BER of GMSK modulation system in AWGN channel and fading channels is analyzed; In [2] GMSK modulation properties of GSM system is analyzed, and a lot of experiments are conducted at $920 \mathrm{MHz}$ frequencies which verify the accuracy of the model; In [3] the effects of GMSK and RS coding on DCPD system performance are analyzed; In [4-6] the performance of RS coding, interleaving in AWGN channel with partial band jamming are analyzed. In [7] the noise effect is analyzed through the integration of the filter pass band of the white noise, but the loss of signal caused by the process of filtering is not considered. The fixed frequency work of VHF radio is the research objects, and the filter, GMSK demodulation, RS decoding and interleaving based are modeled. Simulative experiments are done according to the received link. Contents are as follows: The effects of filtering, GMSK demodulation, RS decoding and interleaving are analyzed and the received model is set up in the first section. The comparison with the theoretical model and analysis of the influence of each link are simulated using the control variable method in the second section, and in the third section the important impact of the received SNR is summarized.

\section{System model}

The receiving process of VHF radio generally include the band-pass filtering, GMSK demodulation, RS decoding and interleaving. Through the analysis of the effect of the BPF filter, GMSK demodulation, RS decoding and de interleaving, the received model is established. The receiving process is as follows:

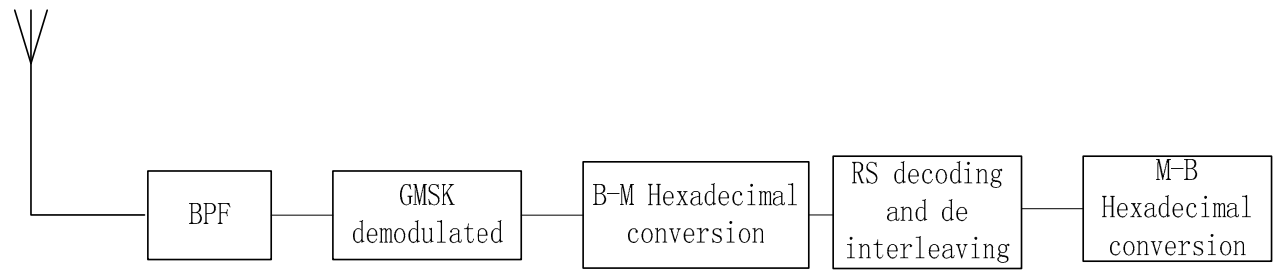

Fig 1 VHF radio receiving process

Performance analysis of BPF filter. The received signal of VHF radio is filtered through the band-pass filter firstly to filter the out-band noise and keep the useful signal, which has been assumed to be the ideal filter to simple the effect of the received SNR[3-6, 8] before. But the actual 
filter attenuation effect on the useful SNR is influenced largely. Through the analysis of the VHF radio technology system, the received process of filtering effect can equal to a Chebyshev I type band-pass filter.

The amplitude frequency response of the filter of Chebyshev I type low-pass filter is

$$
|H(\omega)|=\sqrt{\frac{1}{1+\varepsilon^{2} T_{n}^{2}\left(\frac{\omega}{\omega_{c}}\right)}}, 0<\varepsilon<1
$$

Where $\varepsilon$ is the coefficient of fluctuation deciding the pass-band fluctuation size; $\omega_{c}$ is the pass band cut-off frequency; $T_{n}(\omega)$ is the polynomial of $\mathrm{n}$ order Chebyshev, defined as

$$
T_{n}(\omega)=\left\{\begin{array}{c}
\cos \left(n \cdot \cos ^{-1}(\omega)\right),|\omega| \leq 1 \\
\operatorname{ch}\left(n \cdot \operatorname{ch}^{-1}(\omega)\right),|\omega|>1
\end{array}\right.
$$

Where, $\operatorname{ch}(x)=\frac{e^{x}+e^{-x}}{2}, \quad c h^{-1}(x)=\ln \left(x \pm \sqrt{x^{2}-1}\right)$ 。

The influence caused by Chebyshev filters on SNR is representative by two factors: amplitude frequency response characterization of filters $|H(\omega)|$ and the integration of filter bandwidth of B. $|H(\omega)|$ effects the useful signal power after the filter, B effects the noise power after the received signal after the filter.

Filter bandwidth of B can be defined as the integral:

$$
B=\int_{\omega_{s l}}^{\omega_{s h}}|H(\omega)| d \omega
$$

Where $\omega_{s h}$ and $\omega_{s l}$ are the cut-off frequency of band-pass filter.

The transformation method of pass filter and low pass filter is

$$
\left\{\begin{array}{c}
\omega_{L}=\frac{\omega_{B}^{2}-\omega_{0}^{2}}{B \cdot \omega_{B}} \\
B=\omega_{B 2}-\omega_{B 1} \\
\omega_{0}=\sqrt{\omega_{B 1} \cdot \omega_{B 2}}
\end{array}\right.
$$

Where $\omega_{L}$ and $\omega_{B}$ respectively represent the frequency variable of low-pass and band-pass filters, $\omega_{B 2}$ and $\omega_{B 1}$ respectively represent the higher and lower cut-off frequency of a band pass filter.

The effect caused by the filter on the received signal can equal to the power attenuation of useful signal and the integral of white noise after band pass filter, the SNR is expressed as

$$
\gamma_{B P F}=\frac{|H(\omega)| S(\omega)}{N_{0} B}
$$

Where $S(\omega)$ is the signal power in the frequency of $\omega, N_{0}$ is the noise power spectrum density.

Performance analysis of GMSK demodulation. GMSK modulation is a modulation mode that the base-band signal firstly goes through the Gauss low-pass filter, and then goes through the MSK modulation. So that the power spectrum is relatively concentrated which can effectively reduce the influence of the adjacent channel. Frequency characteristics of the Gauss low-pass filters are

$$
H(f)=\exp \left[-\left(\frac{\ln 2}{2}\right) \cdot\left(\frac{f}{B_{s}}\right)^{2}\right]
$$

In engineering application, $B_{s}$ is the $3 \mathrm{~dB}$ filter bandwidth.

The specified frequency band width of VFH communication system is narrow, mostly using the GMSK modulation and non coherent demodulation [9]. In the channel of white noise, the performance of anti noise ability of GMSK modulation is similar with MSK, the BER of non 
coherent demodulation method of GMSK in AWGN channels[10] is

$$
P_{e, G M S K}=\frac{1}{2} e^{-\frac{E_{b}}{2 N_{0}}}
$$

Where $E_{b}$ is the signal symbol power, $N_{0}$ is the noise power spectrum density.

Simultaneous (5) (6) (7), the BER gone through BPF filter and GMSK demodulation can be obtained

$$
P_{e}=\frac{1}{2} e^{-\frac{|H(\omega)| \cdot S(\omega)}{2 N_{0} \cdot B_{s}}}
$$

Before the RS decoding and interleaving, M binary to hexadecimal converses firstly. Only considering a bit error, the transformation formula of the SER and BER is

$$
P_{s}=\frac{M}{2(M-1)} P_{e}
$$

Where, $P_{s}$ is the symbol error rate, $P_{e}$ is the bit error rate.

Performance analysis of RS decoding and interleaving. $\mathrm{RS}$ code is a multi-level $\mathrm{BCH}$ cyclic block code mode, with strong error correcting ability. VHF radio with RS coding, against unexpected error caused by the fading and interference of the channel. For $R S(n, k), \mathrm{n}$ the number of symbol in a code block, $\mathrm{k}$ represents the number of message symbol. For $\mathrm{m}$ bit encoder, a symbol alphabet of $M=2^{m}$ can be produced. Minimum code distance $d_{\min }=n-k+1$, can correct less than or equal to a symbol error of t. The SER of RS coding system [10] is

$$
P_{S, \mathrm{RS}} \approx \frac{1}{n} \sum_{j=t+1}^{n} j\left(\begin{array}{l}
n \\
j
\end{array}\right) P_{s}^{j}\left(1-P_{s}\right)^{n-j}
$$

Where $P_{s}$ is the SER of modulation channel.

At the same time, VHF radio with packet for reading list of interleaving mode so that burst error produced in the original channel can be transited into a random memory-less independent errors. Interleaving mode of $(p, q)$, divides into $\mathrm{P}$ packet length column $\mathrm{Q}$, constitutes an interleaving depth of interleaving matrix Q. Applied to the coding system, it changes to $R S(q n, \mathrm{q} k)$ code [6], which can correct up to qt burst error symbol, then the SER formula of (10) is

$$
P_{S, \mathrm{RS}+\mathrm{PQ}} \approx \frac{1}{q n} \sum_{j=q t+1}^{q n} j\left(\begin{array}{c}
q n \\
j
\end{array}\right) P_{s}^{j}\left(1-P_{s}\right)^{q n-j}
$$

In radio actually receiving process, a $\mathrm{M}$ hex symbols for binary bit output is converted, in application (9), available BER is

$$
P_{e, \text { final }}=\frac{2(M-1)}{M} P_{S, R S+P Q}
$$

In summary, combined with (6) (8) (9) (11) (12), the received model of VHF radio is established. In the circumstances of component known at the received end, the BER performance of the radio can be done.

\section{The simulation of received link}

For the analysis of the BER influence caused by the filtering and the RS coding and interleaving link, using control variable method, the simulation is done where BER of this model and the rate of the two links of the theoretical model are compared. And the correlation analysis is carried on. Fig. 2 is the BER comparison of this model and the filter link theoretical model, Fig. 3 the BER comparison of this model and RS coding and interleaving sectors of theory model. 


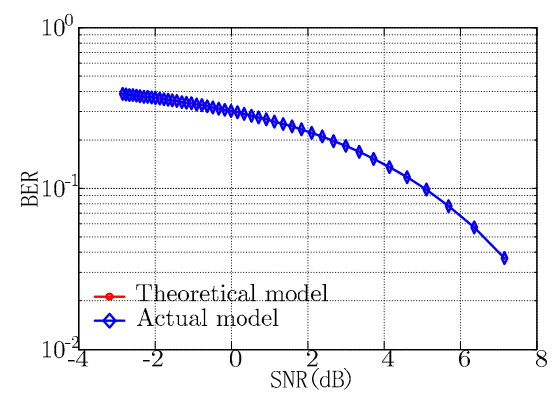

Fig. 2 the BER comparison of this model and the filter link theoretical model

The main factor effecting the BER is the received end of the demodulator filter. For the simulation results, we can see that the theoretical curve and the actual results of filtering curve coincide completely. So that the front-end filter can be regarded as the ideal filter which effect the BER.

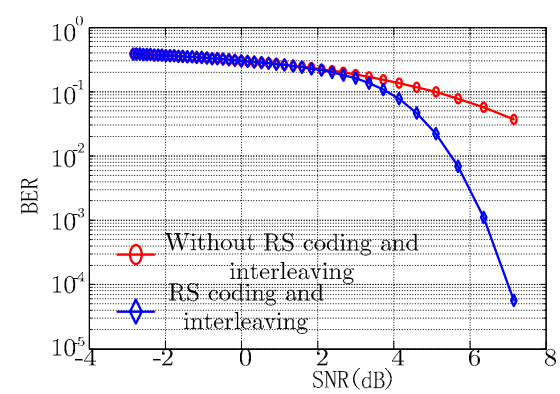

Fig. 3 the BER comparison of this model and model without RS coding and interleaving sectors

In Fig 3, whether there are RS code and interleaving in the system is a big impact on the received BER, and the SNR is larger, the impact effect more obviously. The effect caused by RS code and interleaving system which can correct mistake is exponential growth, consistent with the theoretical analysis in section 1 .

\section{Conclusion}

With the analytical and simulative results of the received link of VHF radio, it can be concluded that:

1. the influence of the filter, mainly concentrate in the final stage filter, namely the baseband filter of demodulation. The front-end filter will affect the SNR, but for the BER of demodulation has little effect.

2. the influence of RS decoding and interleaving is huge, and the effect grows exponentially. When the SNR is large, the effect of error correction is obvious.

As for the analysis for the received performance of VHF radio, the analysis of the demodulation system and RS decoding and interleaving system can be concentrated on.

\section{References}

[1] Ghnimi S, Rajhi A, Gharsallah A. BER perfermance of GMSK modulation under radio mobile propagation environments[C]//Mediterranean Microwave Symposium (MMS), 2011 11th. IEEE, 2011: 305-308.

[2] Miki T, Hatay M. Performance of $16 \mathrm{kbit} / \mathrm{s}$ GMSK transmission with postdetection selection diversity in land mobile radio[J]. Vehicular Technology, IEEE Transactions on, 1984, 33(3): 128-133.

[3] Kamali B, Brinkley R L. Reed-Solomon coding and GMSK modulation for digital cellular packet data systems[C]//Communications, Computers and Signal Processing, 1997. 10 Years PACRIM 1987-1997-Networking the Pacific Rim. 1997 IEEE Pacific Rim Conference on. IEEE, 1997, 2: 745-748.

[4] Lee J S, French R H, Miller M E. Error-correcting codes and nonlinear diversity combining against worst case partial-band noise jamming of frequency-hopping MFSK systems[J]. 
Communications, IEEE Transactions on, 1988, 36(4): 471-478.

[5] Teh K C, Xian W. Performance analyses of Reed-Solomon coded FFH/BFSK linear-combining receiver over multitone jamming and AWGN channels[C]/MILCOM 2000. 21st Century Military Communications Conference Proceedings. IEEE, 2000, 1: 149-153.

[6] YANG Guang,ZHOUJing-lun,LUO Peng-cheng ,Performance Analysis of JTIDS with Partial Band Noise Interference[J]. JOURNALOF NATIONAL UNIVERSITYOF DEFENSE TECHNOLOGY, 2010, 32(1): 122-126.

[7] Zhao Bo, Quan Houde, Cui Peizhang. Quantitative analysis on radio co-site interference[J], Chinese Journal of Radio Science. 2011, 26(5): 837-843.

[8] Han Y, Teh K C. Error probabilities and performance comparisons of various FFH/MFSK receivers with multitone jamming[J]. IEEE transactions on communications, 2005, 53(5): 769-772.

[9] Yan Yunbin, Quan Houde, Cui Peizhang. Simulation and Analysis of Jamming Pattern in GMSK Frequency-Hopping Radio[J]. Computer Measurement \& Control. 2011.19(12):3082-3084

[10]A.Bruce Carlson, Paul B. Crilly. Communication Systems: An Introduction to Signals and Noise in Electrical Communication(Fifth Edition)[M]. Tsinghua University press, 2011. 